\title{
Posição canguru em recém-nascidos pré-termo de muito baixo peso: estudo descritivo
}

Samilly Rodrigues Farias ${ }^{1}$, Flávia de Souza Barbosa Dias ${ }^{2}$, Juliana Bastoni da Silva ${ }^{3}$, Ana Lidia de Lucca Ribeiro Cellere ${ }^{4}$, Lidia Beraldo ${ }^{5}$, Elenice Valentim Carmona ${ }^{6}$

\author{
${ }^{1}$ Enfermeira. Enfermeira Assistencial do \\ Hospital Professor Doutor José Aristodemo \\ Pinotti da Universidade Estadual de \\ Campinas. Campinas, SP, Brasil. E-mail: \\ samillyfarias@gmail.com. \\ ${ }^{2}$ Enfermeira, Mestre em Saúde da Criança \\ e do Adolescente. Discente do Programa \\ de Pós-Graduação em Enfermagem, nível \\ Doutorado, da Universidade Estadual de \\ Campinas. Campinas, SP, Brasil. E-mail: \\ flaviabdias@gmail.com. \\ ${ }^{3}$ Enfermeira, Doutora em Enfermagem na \\ Saúde do Adulto. Professora Doutor da \\ Faculdade de Enfermagem da Universidade \\ Estadual de Campinas. Campinas, SP, \\ Brasil. E-mail: jbastoni@unicamp.br. \\ ${ }^{4}$ Enfermeira. Enfermeira Assistencial da \\ UTI Neonatal do Hospital Estadual de \\ Sumaré. Sumaré, SP, Brasil. E-mail: \\ ana_cellere@yahoo.com.br. \\ ${ }^{5}$ Enfermeira. Supervisora da UTI Neonatal \\ do Hospital Estadual de Sumaré. Sumaré, \\ SP, Brasil. E-mail: \\ lidiaberaldo@yahoo.com.br. \\ ${ }^{6}$ Enfermeira, Doutora em Enfermagem. \\ Professora Doutor da Faculdade de \\ Enfermagem da Universidade Estadual de \\ Campinas. Campinas, SP, Brasil. E-mail: \\ elenicevalentim@uol.com.br.
}

Recebido: 10/11/2015

Aceito: 30/11/2016.

Publicado: 12/06/2017.

Como citar esse artigo:

Farias SR, Dias FSB, Silva JB, Cellere ALLR, Beraldo L, Carmona EV. Posição canguru em recém-nascidos pré-termo de muito baixo peso: estudo descritivo. Rev. Eletr. Enf. [Internet]. 2017 [acesso em: ____19:a15. Disponível em: http://dx.doi.org/10.5216/ree.v19.38433.

\section{RESUMO}

Descrever o número de períodos em que recém-nascidos pré-termo de muito baixo peso estiveram em posição canguru durante internação na unidade neonatal e buscar relações entre variáveis maternas e neonatais com a realização da posição canguru. Estudo descritivo retrospectivo com todos os recém-nascidos pré-termo admitidos em 2012, com peso de nascimento igual ou inferior a $1500 \mathrm{~g}$ e idade gestacional menor que 31 semanas, classificados como pacientes de maior risco clínico, segundo o Clinical Risk Index for Babies. Foram investigados 38 sujeitos, cujo período de hospitalização variou entre 18 a 136 dias. O início da posição canguru ocorreu em média aos 30,8 dias de vida pós-natal $(D P=18,5)$ e a quantidade de períodos em que estiveram em posição canguru foi em média 10,7 vezes. A ocorrência da posição canguru foi menos frequente que as oportunidades advindas da presença materna e esteve relacionado ao maior oferecimento de leite em livre demanda.

Descritores: Método Canguru; Prematuro; Recém-Nascido de muito Baixo Peso; Cuidados de Enfermagem; Enfermagem Neonatal.

\section{INTRODUÇÃO}

Em todo o mundo, nascem cerca de 15 milhões de prematuros a cada ano, sendo que o Brasil se encontra entre os 10 primeiros países onde ocorre maior número desses nascimentos. O baixo peso ao nascimento, associado à prematuridade, é responsável por cerca de 60 a $80 \%$ das mortes neonatais. Além da mortalidade neonatal, outro importante desfecho é o comprometimento no desenvolvimento 
neurológico das crianças sobreviventes ${ }^{(1-2)}$.

O uso de tecnologias complexas e especializadas na assistência ao recém-nascido pré-termo (RNPT) e recém-nascido $(\mathrm{RN})$ de baixo peso e muito baixo peso (peso de nascimento inferior a 2.500 e $1.500 \mathrm{~g}$ respectivamente) têm possibilitado a diminuição dos índices de mortalidade perinatal e neonatal. No entanto, a manutenção da vida do pequeno paciente implica em hospitalização e assistência intensiva, que pode se prolongar por vários dias ou meses, prejudicando o processo natural de apego entre pais e filhos ${ }^{(3)}$.

Para tentar minimizar os efeitos negativos causados por essa separação, o Ministério da Saúde vem implantando a política de atenção humanizada ao RN de baixo peso, denominada Método Canguru, definido como um modelo assistencial perinatal voltado para a humanização que reúne estratégias de intervenção biopsicossocial, sendo seu principal componente o contato pele a pele em posição canguru. Esta posição consiste em acomodar o RN verticalmente, sem roupas, junto ao peito dos pais ou dos familiares, a fim de promover o contato pele a pele em RN de baixo peso e/ou pré-termo ${ }^{(4)}$.

A implantação completa do Método Canguru consiste em três etapas sequenciais, a primeira e a segunda acontecem em ambiente hospitalar e a terceira no domicílio. Na primeira etapa, dentro da unidade de terapia intensiva neonatal (UTIN), é realizado o acolhimento da família e o primeiro contato dos pais com o filho, culminando com o posicionamento canguru. A segunda etapa ocorre fora da UTIN, em uma unidade de internação específica, denominada unidade canguru, onde a mãe é convidada a retornar ao hospital e prestar os cuidados integrais ao seu filho sob supervisão e orientação da equipe de saúde. A terceira etapa ocorre após a alta hospitalar precoce, onde o RN permanece em posição canguru em sua casa na maior parte do $\operatorname{dia}^{(4)}$.

A utilização do Método Canguru (MC) está listada como uma das três intervenções prioritárias mais eficientes no atendimento ao RNPT. Evidências na redução da morbimortalidade e sepse, verificadas a partir de revisões sistemáticas, recomendam o uso do MC em RNPT em países com média e baixa renda ${ }^{(5-6)}$.

A posição canguru está associada à promoção do aleitamento materno, contribuindo com a ampliação do período de amamentação e com o aumento do volume de leite produzido. Além disso, existem evidências de que esta posição melhora o vínculo entre mãe e filho e promove a participação dos pais nos cuidados com o RN, fortalecendo o desempenho dos papéis de pai e mãe. Do mesmo modo, a posição canguru favorece uma melhor regulação térmica e estabilidade fisiológica, estimula o desenvolvimento neurocomportamental e traz benefícios para o sono e alívio da dor do RNPT ${ }^{(7-9)}$.

A posição Canguru deve ser encorajada pelos profissionais que atuam em UTIN e ocorrer de forma que seja prazerosa e estimulante para a formação dos laços afetivos entre pais e filho, provendo calor, amor e estímulos por um período de tempo em que ambos sintam-se bem ${ }^{(10)}$. Dada sua importância em RNPT na redução da morbimortalidade e promoção do aleitamento materno, dentre outros benefícios, as unidades neonatais devem promover esta estratégia, registrando-a de forma a acompanhar sua implementação e resultados maternos e neonatais. Assim, este artigo teve como objetivo descrever o número de períodos em que RNPT, com peso abaixo de 1500g, estiveram em posição canguru ao longo da internação e buscar 
relações entre variáveis maternas e neonatais com a realização da posição Canguru.

\section{MÉTODO}

Trata-se de um estudo descritivo, com coleta de dados retrospectiva, que foi desenvolvido a partir de dados de prontuários de recém-nascidos que foram admitidos em UTIN durante o ano de 2012. A unidade neonatal em questão possui 12 leitos de terapia intensiva, 10 leitos semi-intensivos e dois leitos de unidade canguru e está inserida em um Hospital Amigo da Criança, de caráter público de ensino, assistência e pesquisa na cidade de Sumaré, Estado de São Paulo. O hospital é administrado por uma Universidade Estadual e mantido com recursos do SUS.

Fizeram parte desta investigação os prontuários de todos os RNPT admitidos durante o ano de 2012, considerados como pacientes de maior risco clínico, segundo o escore Clinical Risk Index for Babies (CRIB). Esse índice de gravidade é útil para avaliar o risco de mortalidade segundo as variáveis peso de nascimento, idade gestacional, presença de malformações congênitas, excesso de base e fração inspirada de oxigênio. Cada um dos itens avaliados recebe uma pontuação cuja somatória pode variar de zero a 23 , sendo os pacientes classificados em quatro graus, onde o maior grau corresponde à maior gravidade ${ }^{(11)}$.

Os critérios de inclusão para o estudo foram: peso de nascimento menor ou igual a $1500 \mathrm{~g}$, idade gestacional menor que 31 semanas e ter nascido no hospital onde foi realizada a pesquisa. Os critérios de exclusão foram: malformações incompatíveis com a vida; óbitos ocorridos antes de 12 horas de vida e RN que evoluíram para óbito antes que pudesse ser realizado o primeiro posicionamento canguru.

O presente estudo foi aprovado pelo Comitê de Ética em Pesquisa da Faculdade de Ciências Médicas da UNICAMP sob o no 320.981. Foi obtida a dispensa da utilização do Termo de Consentimento Livre Esclarecido, visto que, a pesquisa foi realizada com dados de prontuários, sem necessidade de contato com os sujeitos do estudo.

Os prontuários foram localizados no Serviço de Arquivo Médico e Estatística (SAME) do referido hospital, onde foram coletados os dados e, posteriormente, registrados em uma planilha eletrônica. Para a extração de dados dos prontuários elaborou-se um instrumento contendo variáveis sociodemográficas e clínicas, maternas e neonatais. Em relação às variáveis maternas buscou-se as seguintes informações: idade, paridade, situação conjugal, tipo de parto, uso de corticoide pré-natal, acompanhamento pré-natal, número de visitas. Quanto às variáveis neonatais foram coletadas sexo, peso de nascimento, crescimento intrauterino, Apgar do 1ㅇ e 5o minuto, presença de distúrbio respiratório, dias de hospitalização, peso e idade no primeiro dia em posição canguru, classificação segundo grau CRIB, tipo de leite, número de vezes que recebeu leite materno e via de alimentação na alta hospitalar. Também foi registrado o número de oportunidades para estar em posição Canguru e de períodos em que ocorreu a posição Canguru.

Assumiu-se como oportunidade para estar em posição Canguru, os períodos (manhã, tarde ou noite) em que o RN apresentava-se clinicamente apto, sendo possível até três oportunidades diárias. Na unidade onde foi realizado o estudo, o RNPT recebe prescrição médica para posição Canguru após avaliação de sua 
condição clínica, considerando ganho de peso adequado e progressivo, estabilidade térmica, padrão respiratório e ausência de episódios de apnéia.

A análise dos dados foi feita no programa Statistical Analysis System, versão 9.2 e analisados segundo estatística descritiva e inferencial. Para descrever o perfil demográfico e clínico da amostra foram construídas tabelas de frequência absoluta $(\mathrm{n})$ e relativa (\%).

As correlações entre as variáveis quantitativas foram avaliadas por meio do coeficiente de correlação de Spearman. Este coeficiente não paramétrico varia de -1 a 1 . Considerou-se seguinte classificação do coeficiente de correlação: 0,1 a 0,29 (fraca), 0,30 a 0,49 (moderada), $\geq 0,50$ (forte) ${ }^{(12)}$. As comparações entre as variáveis categóricas e quantitativas foram realizadas por meio do teste não paramétrico de MannWhitney. Para todas as análises foi considerado um nível de significância de 5\%.

\section{RESULTADOS}

Durante o ano de 2012 foram admitidos nesta unidade 46 RN com peso menor ou igual a $1500 \mathrm{~g}$ e/ou idade gestacional inferior a 31 semanas, que nasceram neste hospital. Foram excluídos do estudo oito RN que foram a óbito antes de iniciarem a posição Canguru, sendo assim, fizeram parte da amostra 38 sujeitos. No grupo que evoluiu para óbito o tempo médio de vida foi de 8,4 dias ( $\mathrm{DP}=14,2)$ com peso médio de nascimento de 602,5g (DP=215,4).

O peso de nascimento dos sujeitos variou de 650g a 1.500g, com média de 1.096g ( $\mathrm{DP}=217$ ) e mediana de 1.092g. O período de hospitalização variou de 18 a 136 dias, com mediana de 53 dias. A média de peso dos RN na alta hospitalar foi de $2.285 \mathrm{~g}$, com mediana $2.162 \mathrm{~g}$, peso mínimo de $1.875 \mathrm{~g}$ e máximo de $4.630 \mathrm{~g}$. A Tabela 1 traz outros dados relacionados aos RNPT. 
Tabela 1: Caracterização demográfico-clínica dos recém-nascidos pré-termo de muito baixo peso. Sumaré, SP, Brasil, 2012.

\begin{tabular}{|c|c|c|c|}
\hline & Variável & $\mathbf{n}$ & $\%$ \\
\hline \multicolumn{4}{|l|}{ Sexo } \\
\hline Masculino & & 15 & 39,5 \\
\hline Feminino & & 23 & 60,5 \\
\hline \multicolumn{4}{|l|}{ Crescimento intrauterino* } \\
\hline PIG & & 17 & 47,2 \\
\hline AIG & & 19 & 52,8 \\
\hline Sem informação & & 2 & \\
\hline \multicolumn{4}{|c|}{ Apgar no primeiro minuto de vida } \\
\hline$>7$ & & 25 & 65,8 \\
\hline$<7$ & & 13 & 34,2 \\
\hline \multicolumn{4}{|c|}{ Apgar no quinto minuto de vida } \\
\hline$>7$ & & 36 & 94,7 \\
\hline$<7$ & & 2 & 5,3 \\
\hline \multicolumn{4}{|c|}{ Distúrbio respiratório ao nascimento } \\
\hline Sim & & 33 & 89,2 \\
\hline Não & & 4 & 10,8 \\
\hline Sem informação & & 1 & \\
\hline \multicolumn{4}{|l|}{ Classificação grau CRIB** } \\
\hline 1 & & 28 & 73,7 \\
\hline 2 & & 8 & 21,0 \\
\hline 3 & & 2 & 5,3 \\
\hline \multicolumn{4}{|c|}{ Tipo de alimentação descrita na alta hospitalar } \\
\hline Aleitamento exclusivo & & 11 & 29,7 \\
\hline Aleitamento misto & & 18 & 48,6 \\
\hline Aleitamento artificial & & 8 & 21,6 \\
\hline Sem informação & & 1 & \\
\hline \multicolumn{4}{|c|}{ Leite materno*** no último dia de hospitalização } \\
\hline > seis vezes & & 9 & 23,7 \\
\hline$<$ seis vezes & & 17 & 44,7 \\
\hline Nenhuma vez & & 12 & 31,6 \\
\hline \multicolumn{4}{|c|}{ Via de oferecimento do leite no último dia de hospitalização } \\
\hline Sonda & & 1 & 2,6 \\
\hline Sonda e via oral & & 3 & 7,9 \\
\hline Oral & & 34 & 89,5 \\
\hline
\end{tabular}

* Crescimento intrauterino: PIG = Pequeno para a Idade Gestacional e AIG = Adequado para a Idade Gestacional

** Clinical Risk Index for Babies: grau 1 para escores de 0 a 5, grau 2 de 6 a 10, grau 3 de 11 a 15, e grau 4 para pontuações maiores do que 15.

*** Leite materno cru e/ou pasteurizado.

A média de idade das mães foi de 26,8 anos ( $D P=7,4)$, variando de 19 a 41 anos. Quanto ao número de gestações, a amostra apresentou uma mediana de duas gestações, variando de uma a oito gestações. Outros aspectos da caracterização das mães são apresentados na Tabela 2. 
Tabela 2: Caracterização demográfico-clínica das mães dos recém-nascidos pré-termo de muito baixo peso. Sumaré, SP, Brasil, 2012.

\begin{tabular}{|c|c|c|c|}
\hline & Variável & $\mathbf{n}$ & $\%$ \\
\hline \multicolumn{4}{|l|}{ Cidade de procedência } \\
\hline Sumaré & & 24 & 64,8 \\
\hline Outras & & 13 & 35,2 \\
\hline Sem informação & & 1 & \\
\hline \multicolumn{4}{|l|}{ Situação conjugal } \\
\hline Com companheiro & & 6 & 60 \\
\hline Sem companheiro & & 4 & 40 \\
\hline Sem informação & & 28 & \\
\hline \multicolumn{4}{|l|}{ Parto } \\
\hline Vaginal & & 10 & 27,1 \\
\hline Cesárea & & 27 & 72,9 \\
\hline Sem informação & & 1 & \\
\hline \multicolumn{4}{|c|}{ Uso de corticoides pré-natal } \\
\hline Sim & & 13 & 38,2 \\
\hline Não & & 21 & 61,8 \\
\hline Sem informação & & 4 & \\
\hline \multicolumn{4}{|c|}{ Acompanhamento pré-natal } \\
\hline UBS* & & 17 & 70,8 \\
\hline Unidade referenciada & & 6 & 25 \\
\hline Não & & 1 & 4,2 \\
\hline Sem informação & & 14 & \\
\hline
\end{tabular}

* UBS: Unidade Básica de Saúde

A Tabela 3 apresenta a descrição de como ocorreu a posição canguru entre mães e filhos, considerando períodos em que este contato ocorreu.

Tabela 3: Caracterização da posição canguru em recém-nascidos pré-termo de muito baixo peso hospitalizados em Unidade de Terapia Intensiva Neonatal. Sumaré, SP, Brasil, 2012.

\begin{tabular}{cccccc}
\hline Variável & Média & DP & Mínimo & Mediana & Máximo \\
\hline Número de períodos em que a mãe esteve na unidade & 66,5 & 35,1 & 0 & 59,5 & 178 \\
Número de períodos em que ficou em posição Canguru & 10,7 & 8,7 & 0 & 8 & 39 \\
Número de períodos em que poderia ter estado em posição Canguru* & 101,6 & 49,8 & 18 & 87 & 213 \\
Peso em gramas quando RN ficou em posição Canguru pela primeira vez & 1520 & 305,4 & 1060 & 1490 & 2490 \\
Dias de vida quando RN ficou em posição Canguru pela primeira vez & 30,8 & 18,5 & 4 & 25 & 69 \\
\hline
\end{tabular}

* RNPT clinicamente apto para posição canguru.

Os dias de vida em que os prematuros foram colocados para sucção em mama materna pela primeira vez variaram de quatro dias de vida a 85 , com mediana de 46 . Já o peso de início deste processo variou de $1.220 \mathrm{~g}$ a $2.480 \mathrm{~g}$, com mediana de $1.745 \mathrm{~g}$.

$\mathrm{Na}$ Tabela 4 verificou-se que houve forte correlação positiva entre os dias de vida em que se iniciou a posição canguru e os dias de hospitalização. Também houve forte correlação negativa entre os dias de vida em que se iniciou a posição canguru e o peso de nascimento. 
Tabela 4: Correlação entre dados da caracterização da posição canguru e variáveis maternas e neonatais ( $n=38)$. Sumaré, SP, Brasil, 2012.

\begin{tabular}{|c|c|c|c|c|c|}
\hline & $\begin{array}{c}\text { Idade } \\
\text { materna }\end{array}$ & $\begin{array}{l}\text { Número de } \\
\text { gestações }\end{array}$ & $\begin{array}{c}\text { Dias de } \\
\text { hospitalização }\end{array}$ & $\begin{array}{c}\text { Peso de } \\
\text { nascimento }\end{array}$ & $\begin{array}{l}\text { CRIB } \\
\text { grau }\end{array}$ \\
\hline Períodos de presença materna & 0,30 & $-0,11$ & 0,58 & $-0,47$ & 0,14 \\
\hline ( $p$ valor) & $(0,06)$ & $(0,5)$ & $(0,0001)$ & $(0,002)$ & $(0,3)$ \\
\hline Peso ao iniciar posição Canguru* & 0,13 & 0,17 & 0,19 & 0,18 & 0,21 \\
\hline ( $p$ valor) & $(0,4)$ & $(0,3)$ & $(0,3)$ & $(0,3)$ & $(0,2)$ \\
\hline Dias de vida ao iniciar posição canguru* & 0,05 & 0,09 & 0,80 & $-0,60$ & 0,45 \\
\hline$(p$ valor $)$ & $(0,8)$ & $(0,6)$ & $(<0,0001)$ & $(<0,0001)$ & $(0,005)$ \\
\hline $\begin{array}{l}\text { Oportunidades para estar em posição } \\
\text { canguru* }\end{array}$ & 0,29 & $-0,06$ & 0,66 & $-0,45$ & 0,48 \\
\hline$(p$ valor $)$ & $(0,08)$ & $(0,7)$ & $(<0,0001)$ & $(0,005)$ & $(0,002)$ \\
\hline Períodos em posição Canguru & $-0,02$ & $-0,35$ & 0,11 & $-0,18$ & 0,01 \\
\hline ( $p$ valor) & $(0,8)$ & $(0,03)$ & $(0,5)$ & $(0,2)$ & $(0,9)$ \\
\hline
\end{tabular}

Também se buscou investigar possíveis associações entre os dados relacionados à posição Canguru, anteriormente citados, e variáveis maternas e neonatais, como: tipo de parto; uso de corticoide ante-natal; sexo do bebê; crescimento intrauterino, dentre outros, porém não foram encontradas associações significativas.

Quanto antes a posição Canguru foi iniciada, considerando-se peso e dias de vida pós-natal em que ela se deu pela primeira vez, houve maior oferecimento de leite, materno e/ou artificial, em livre demanda, no último dia de internação, segundo o teste de comparação de Mann-Whitney $(p=0,01)$.

\section{DISCUSSÃO}

O RN de baixo peso ao nascer e prematuros frequentemente precisam de assistência neonatal especializada, chegando a apresentar maior risco de mortalidade e morbidade ao longo de todo o primeiro ano de vida. A quantidade expressiva de partos cesárea (72,9\%), nesta amostra, indica que tais gestações apresentavam risco, sendo necessária a intervenção cirúrgica para manutenção da vida da mãe e/ou do feto, o que geralmente se relaciona à necessidade de cuidados intensivos para o RN devido à prematuridade $\mathrm{e}$ complicações relacionadas ${ }^{(1,13)}$.

Dentre as complicações relacionadas à prematuridade, observamos que nesta amostra destacam-se os distúrbios respiratórios. Para diminuir a incidência de problemas respiratórios ao nascimento, o uso de corticoide pré-natal é recomendado para todas as gestantes entre 24 e 34 semanas com risco de parto prematuro $^{(14)}$. Neste estudo, foi possível verificar que $61,8 \%$ das gestantes não receberam o corticoide prénatal, o que pode se relacionar ao maior risco de complicações respiratórias para os bebês, evidenciando uma lacuna na assistência à saúde dessas gestantes. Tais complicações respiratórias podem representar uma condição clínica inicial, que pode ser revertida, mas podem também evoluir para um distúrbio mais grave, potencialmente letal, o que faz com que aumente o período de permanência do RN em unidades de cuidado intensivo $^{(14)}$.

Quanto ao acompanhamento pré-natal, não se tem informação a respeito de 14 mães (36,8\% de 38), 
ao passo que 17 mães (70,8\%), das quais se obteve a informação, o realizaram na Unidade Básica de Saúde. A realização deste acompanhamento é uma das principais ações de promoção à saúde da gestante e do feto, visto que auxilia a prevenir eventos adversos na gestação e nascimento. A melhoria das condições de realização desta prática têm possibilitado a redução dos determinantes da morbimortalidade perinatal, refletindo uma importante meta alcançada na atenção básica ${ }^{(15)}$.

Considerando os dados apresentados na Tabela 1, verifica-se que, apesar dos conhecidos benefícios do aleitamento materno exclusivo, principalmente para os prematuros, sua prevalência na alta hospitalar foi muito baixa na amostra estudada, mesmo com utilização do Método Canguru neste hospital. Este resultado foi ainda inferior ao de um estudo realizado na região Nordeste do Brasil, num hospital de nível terciário e que pratica o Método Canguru, onde $56 \%$ dos prematuros receberam alta hospitalar em aleitamento exclusivo $^{(16)}$. Além disso, a falta de suporte e promoção do aleitamento materno pela equipe de saúde também pode ser um fator contribuinte para esta baixa prevalência. As intervenções da equipe são fundamentais no que se refere a manutenção da lactação enquanto o bebê não está apto a alimentar-se por via oral, assim como o oferecimento de orientações, incentivo e o manejo clínico da amamentação(17).

Embora o relatório de alta hospitalar dos pacientes em questão descreva que o aleitamento materno exclusivo esteve presente para 11 bebês $(29,7 \%)$, os dados do último dia de hospitalização denotam que o leite materno não foi oferecido como alimento exclusivo para alguns deles. Isto pode estar relacionado à ausência da mãe ou de leite materno, visto que a unidade não tem banco de leite, apenas lactário.

A proposta do método Canguru é um atendimento humanizado, de baixo custo para os recém-nascidos de baixo peso, que consiste em promover o contato pele a pele entre mãe e filho o mais precocemente possível, através da posição Canguru, bem como o aleitamento materno e a consequente alta precoce ${ }^{(8)}$. Embora diversos estudos ${ }^{(18-21)}$ evidenciem a segurança e os benefícios da realização precoce da posição Canguru em recém-nascidos de prematuridade extrema e muito baixo peso, na amostra estudada, o início da posição Canguru se deu tardiamente, principalmente naqueles com menor peso de nascimento. Isto leva a questionar se a condição clínica destes pacientes não foi favorável para esta estratégia ou se os critérios adotados pela equipe médica para sua indicação são adequados.

Esse dado reflete uma lacuna na prática do Método Canguru para esta população e pode estar relacionada à falta de treinamento e insegurança dos profissionais que, de acordo com a literatura, muitas vezes, apresentam receio de que ocorra alteração de sinais vitais, mobilização de cateter umbilical ou cateter central de inserção periférica, extubação acidental ou perda de acesso venoso.

A unidade onde foi realizado o estudo permite acesso irrestrito dos pais para visitarem seus filhos, apesar disso, verificamos que o número de períodos em que a mãe esteve presente na unidade ocorreu em apenas 34,7\% dos períodos em que os recém-nascidos poderiam ter recebido visita, considerando o tempo médio de internação. Não foram investigados os motivos da ausência da mãe na unidade, mas pressupõe-se que fatores relacionados à locomoção, dificuldade com quem deixar os outros filhos, necessidade de realização de atividades domésticas e cuidados com outros membros da família, além das questões 
emocionais que envolvem ser mãe de um recém-nascido internado em unidade de terapia intensiva neonatal, possam dificultar a presença materna na unidade.

Considera-se de extrema importância o estímulo, investigação e o registro pela equipe de enfermagem das condições sociais e econômicas que envolvem as famílias dos bebês internados. Os dados também evidenciam que a ocorrência dos períodos em posição canguru foi menos frequente do que as oportunidades existentes, ou seja, os bebês ficaram em contato com as mães em cerca de $10 \%$ dos períodos possíveis.

Não se pode afirmar aqui os motivos que levaram as mães a não estarem com maior frequência em posição Canguru com seus filhos na unidade estudada. Entretanto, as questões maternas, dificuldades e limitações para estar junto ao filho devem fazer parte das avaliações do enfermeiro neonatal e contempladas em seu plano de cuidados ${ }^{(22)}$. Outra investigação pertinente é a atitude da equipe quanto à promoção da posição Canguru, quanto ao reconhecimento do valor desta proposta, em detrimento do trabalho rotineiro e do cumprimento de tarefas específicas. Apesar das conhecidas vantagens desta estratégia, a literatura ainda traz que o método não é amplamente aplicado em unidades neonatais, o que corrobora com estes $\operatorname{achados}^{(23)}$

Verificou-se que quanto menor foi o peso de nascimento do RN, maior foi o número de períodos em que ocorreu a presença materna na unidade, mas isto não significou maior número de períodos em que a posição Canguru ocorreu. Outra associação possível se deu quanto às gestações: quanto maior o número de gestações da mãe, menor foi o número de períodos em que o bebê esteve em posição Canguru. $\mathrm{O}$ que pode estar relacionado à atenção e aos cuidados que a mãe precisa dedicar também aos outros filhos que estão em casa ${ }^{(10)}$. Verificou-se ainda que maiores valores de CRIB estiveram associados ao início mais tardio da posição Canguru ao longo da internação, o que pode indicar que se aguardou a estabilidade clínica para a implementação desta estratégia.

A posição Canguru necessita de uma atenção individualizada e a mãe deve ser estimulada a realizá-lo desde o momento em que o bebê apresenta condições clínicas para tanto. Entretanto, é essencial investigar as dificuldades maternas frente a esta situação, o significado que ela atribui à posição Canguru, esclarecendo a importância para o bebê no que se refere a aspectos psicológicos e fisiológicos, bem como resposta ao tratamento. Por outro lado, a proposta da posição Canguru não pode desconsiderar o desejo da mãe e as questões familiares e pessoais que podem dificultar sua realização ${ }^{(10,13,23)}$.

Como reafirmado na literatura ${ }^{(23-25)}$, o estímulo à presença materna na unidade favorece mais oportunidades para a posição Canguru, assim como todos os benefícios advindos deste contato, como o vínculo e o estabelecimento do aleitamento materno, desde que isto seja apoiado pela equipe de saúde.

Algumas informações importantes para a caracterização das mães não foram localizadas no prontuário. A relevância de tais dados se dá porque poderiam ser direcionadores de ações de enfermagem e de intervenções da equipe de saúde como um todo. Registros incompletos podem trazer impacto negativo na assistência, visto que não se tem como apreender a condição clínica real, bem como os resultados obtidos após assistência prestada. Outro impacto é que isto limita o desenvolvimento de estudos retrospectivos e a 
construção de bancos de dados fidedignos para caracterizar a população atendida, bem como a evolução epidemiológica.

\section{CONCLUSÃO}

De um total de 38 prontuários de RN prematuros nascidos abaixo de $1.500 \mathrm{~g}$, em um hospital público de ensino, verificou-se que a posição Canguru foi promovida, embora de forma menos frequente do que as oportunidades existentes, advindas da presença materna na unidade. Com hospitalizações que duraram de 18 a 136 dias, as mães estiveram com seus filhos em posição Canguru em uma média de 10,6 períodos $(D P=8,7)$. Estes resultados demonstram que embora a literatura aponte inúmeros benefícios do Método Canguru, ele não vem sendo adequadamente implementado na prática clínica. O que requer monitoramento nas unidades neonatais, de forma a compreender os obstáculos para esta implementação, no intuito de elaborar estratégias para alcançar os resultados potenciais do método.

Neste estudo, as mulheres que tinham mais filhos realizaram com menor frequência a posição Canguru com o filho hospitalizado, provavelmente, devido às demandas de cuidados aos outros filhos, além da necessidade de estar no hospital. A posição Canguru foi associada ao maior oferecimento de leite em livre demanda na amostra de recém-nascidos pré-termo do nosso estudo, mas não houve associação com o aleitamento materno exclusivo. Portanto, a realização do cuidado individualizado, que consiste em investigar e considerar necessidades da mãe na assistência neonatal pode auxiliar a identificar características e vivências maternas que limitam a interação com o filho. Isto pode permitir que ela também seja assistida no processo de maternar o filho hospitalizado.

Este estudo teve como limitação a impossibilidade de avaliar as intercorrências ocorridas ao longo do posicionamento, bem como o tempo em horas que cada paciente ficou em posição Canguru junto à mãe, devido à insuficiência dos registros nos prontuários. Verifica-se que a proposta do Método Canguru precisa ser melhor acompanhada e descrita por meio de registros sistematizados e fidedignos, que sirvam para avaliação da assistência prestada, sua continuidade, bem como para fins de pesquisa.

Futuros estudos poderiam investigar o significado desta proposta para a equipe de Enfermagem, visto ser quem mais tem oportunidade para promovê-la. Esta tecnologia de baixo custo precisa ser melhor compreendida e valorizada na assistência neonatal.

\section{REFERÊNCIAS}

1. Lawn JE, Cousens S, Zupan J; Lancet Neonatal Survival Steering Team. 4 million neonatal deaths: when? Where? Why? Lancet. 2005;365(9462):891-900.

2. Lawn JE, Davidge R, Paul VK, von Xylander S, Johnson JG, Costello A, et al. Born Too Soon: Care for the Preterm Baby. Reproductive Health. 2013, 10(Suppl 1):S6.

3. Ministério da Saúde (Brasil). Secretaria de Atenção à Saúde. Departamento de Ações Programáticas e Estratégicas. Atenção à saúde do recém-nascido: guia para os profissionais de saúde. 2 ed. Brasília: Editora do Ministério da Saúde; 2012. 192p. v 1. 
4. Ministério da Saúde (Brasil). Secretaria de Atenção à Saúde. Departamento de Ações Programáticas Estratégicas. Atenção humanizada ao recém-nascido de baixo peso: Método Canguru. 2 ed. Brasília: Editora do Ministério da Saúde; 2012. 204p.

5. March of Dimes, PMNCH, Save the Children, World Health Organization. Born Too Soon: The Global Action Report on Preterm Birth. Howson CP, Kinney MV, Lawn JE, editors. World Health Organization: Geneva, 2012.

6. Conde-Agudelo A, Díaz-Rossello JL. Kangaroo mother care to reduce morbidity and mortality in low birthweight infants. Cochrane Database of Syst Rev. 2014.

7. Nagai S, Yonemoto N, Rabesandratana N, Andrianarimanana D, Nakayama T, Mori R. Long-term effects of earlier initiated continuous Kangaroo Mother Care (KMC) for low-birth-weight (LBW) infants in Madagascar. Acta Paediatr. 2011;100(12):e241-7.

8. Moore ER, Anderson GC, Bergman N, Dowswell T. Early skin to skin contact for mothers and their healthy newborn infants. Cochrane Database of Syst Rev. 2012.

9. Baley J; Committee on Fetus and Newborn. Skin-to-Skin Care for Term and Preterm Infants in the Neonatal ICU. Pediatrics. 2015; 136(3):596-9.

10. Arivabene JC, Tyrrell MAR. Kangaroo Mother Method: mothers' experiences and contributions to nursing. Rev Latino Am Enfermagem. 2010;18(2):262-87.

11. Sarquis ALF, Miyaki M, Cat MNL. The use of CRIB score for predicting neonatal mortality risk. J Pediatr. 2002;78(3):225-9.

12. Cohen J. Statistical power analysis for the behavioral sciences. 2ed. New Jersey: Lawrence Erlbaum Associates; 1988. p.75-108.

13. Almeida H, Venâncio SI, Sanches MTC, Onuki D. The impact of the Kangaroo care on exclusive breastfeeding in low birth weight newborns. J Pediatr. 2010; 86(3):250-253.

14. Brownfoot FC, Gagliardi DI, Bain E, Middleton P, Crowther CA. Different corticosteroids and regimens for accelerating fetal lung maturation for women at risk of preterm birth. Cochrane Database of Syst Rev. 2013.

15. Vettore MV, Dias M, Vettore MV, Leal MC. Assessment of quality of prenatal care among pregnant women with a history of premature deliveries using the Brazilian National Health Service in Rio de Janeiro, Brazil. Rev Bras Saude Mater Infant. 2013;13(2):89-100.

16. Menezes MAS, Garcia DC, Melo EV, Cipolotti R. Preterm newborns at Kangaroo Mother Care: a cohort follow-up from birth to six months. Rev Paul Pediatr. 2014;32(2):171-7.

17. Haroon S, Das JK, Salam RA, Imdad A, Bhutta ZA. Breastfeeding promotion interventions and breastfeeding practices: a systematic review. BMC Public Health. 2013; 13(3): S3-S20.

18. Mörelius E, Angelhoff C, Eriksson J, Olhager E. Time of initiation of skin-to-skin contact in extremely preterm infants in Sweden. Acta Paediatr. 2012;101(1):14-8.

19. Tuoni C, Scaramuzzo RT, Ghirri P, Boldrini A, Bartalena L. Kangaroo Mother Care: four years of experience in very low birth weight and preterm infants. Minerva Pediatr. 2012;64(4):377-83.

20. Maastrup R, Greisen G. Extremely preterm infants tolerate skin-to-skin contact during the first weeks of life. Acta Paediatr. 2010;99(8):1145-9.

21. Azevedo VMGO, Xavier CC, Gontijo FO. Safety of Kangaroo Mother Care in Intubated Neonates Under 1500 g. J Trop Pediatr. 2012;58(1):38-42.

22. Frello AT, Carraro TE. Nursing and the relationship with the mothers of newborns in the Neonatal Intensive Care Unit. Rev Bras Enferm. 2012;65(3):514-21.

23. Gontijo TL, Meireles AL, Malta DC, Proietti FA, Xavier CC. Evaluation of implementation of humanized care to low weight newborns - the Kangaroo Method. J Pediatr. 2010;86(1):33-9.

24. Lawn JE, Mwansa-Kambafwile J, Horta BL, Barros FC, Cousens S. Kangaroo mother care to prevent neonatal deaths due to preterm birth complications. Int J Epidemilogy. 2011; 39(Supp 1):i144-i154.

25. Gontijo TL, Xavier CC, Freitas MIF. Evaluation of the implementation of Kangaroo Care by health administrators, professionals, and mothers of newborn infants. Cad Saúde Pública. 2012;28(5):935-44. 